\title{
FEM analyses of a CVD diamond Brewster window
}

\author{
G. Aiello, G. Grossetti, A. Meier, T. Scherer, S. Schreck, P. Spaeh, D. Strauss, A. Vaccaro
}

Institute for Applied Materials, Karlsruhe Institute of Technology (KIT), P.O. Box 3640,

D-76021 Karlsruhe, Germany

\section{Introduction and background}

Chemical vapor deposition (CVD) diamond windows are a crucial component in heating and current drive $(\mathrm{H} \& \mathrm{CD})$ applications. In order to minimize the amount of reflected power from the diamond disc, its thickness must match the desired beam wavelength, thus proper targeting of the plasma requires movable beam reflectors. This is the case, for instance, of the ITER electron cyclotron H\&CD system. However, looking at DEMO, the higher heat loads and neutron fluxes could make the use of movable parts close to the plasma difficult. The issue might be solved by using gyrotrons able to tune the beam frequency to the desired resonance, but this concept requires transmission windows that work in a given frequency range (e.g. 105-140 GHz), such as the Brewster window. It consists of a CVD diamond disc brazed to two copper cuffs at the Brewster angle. The brazing process is carried out at about $800^{\circ} \mathrm{C}$ and then the temperature is decreased down to room temperature. Diamond and copper have very different thermal expansion coefficients, therefore high stresses build up during the cool down phase that might lead to failure of the disc. Considering also the complex geometry of the window with the skewed position of the disc analyses are required in the first place to check its feasibility. The cool down phase was simulated by FEM structural analyses for several geometric and constraint configurations of the window. A study of an indirectly water-cooled configuration was also performed, considering the power absorption in the diamond disc due to a $\mathrm{HE}_{11}$ mode beam.

\section{Methods}

\section{Reference configuration}

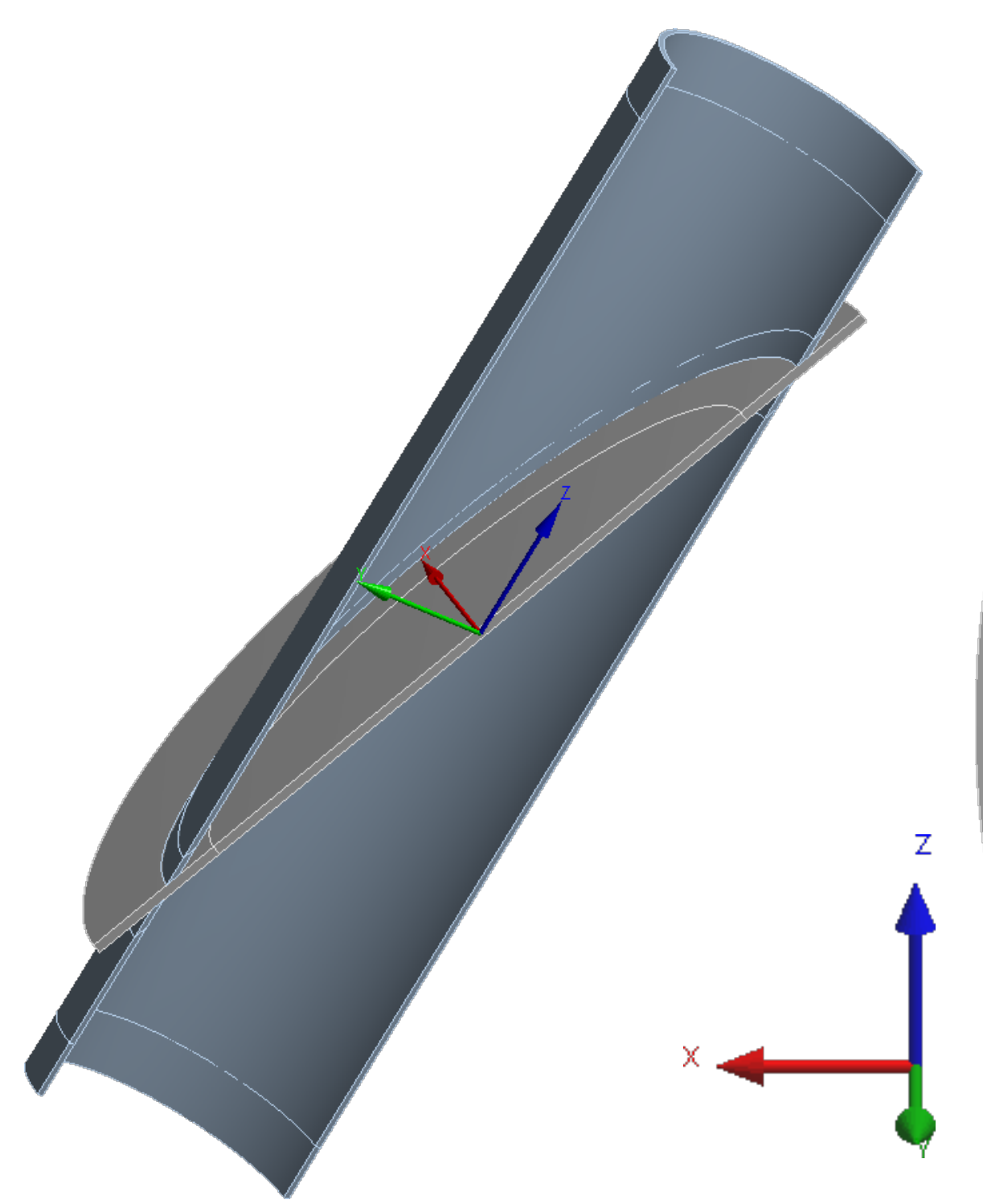

- Elliptical CVD diamond disc $160^{\star} 80 \mathrm{~mm}$ thickness $1.9 \mathrm{~mm}$, Brewster angle $67.22^{\circ}$ - Cylindrical OFHC copper cuffs of inner diameter $50 \mathrm{~mm}$, thickness $1 \mathrm{~mm}$, total length $200 \mathrm{~mm}$

- Symmetry along the disc major axis; - Constant, temperature dependent, non linear material properties:

- Fixed support at the two ends of the cuffs Structural analysis with equilibrium temperature of $800^{\circ} \mathrm{C}$ and applied thermal condition of $20^{\circ} \mathrm{C}$

\section{Geometric and constraint configurations}

- Thickness: from 0.8 to $2 \mathrm{~mm}$ for the cuffs and from 1 to $2.5 \mathrm{~mm}$ for the disc; - Aspect ratio of the disc: 1.4 and 2.33 with major axis of $140 \mathrm{~mm}$ and minor axis respectively of $100 \mathrm{~mm}$ and $60 \mathrm{~mm}$;

- Boundary condition: upper end of the cuffs free to move along the z-axis only (configuration 1) and upper end of the cuffs without constraints (configuration 2).

\section{Indirectly water-cooled configuration}

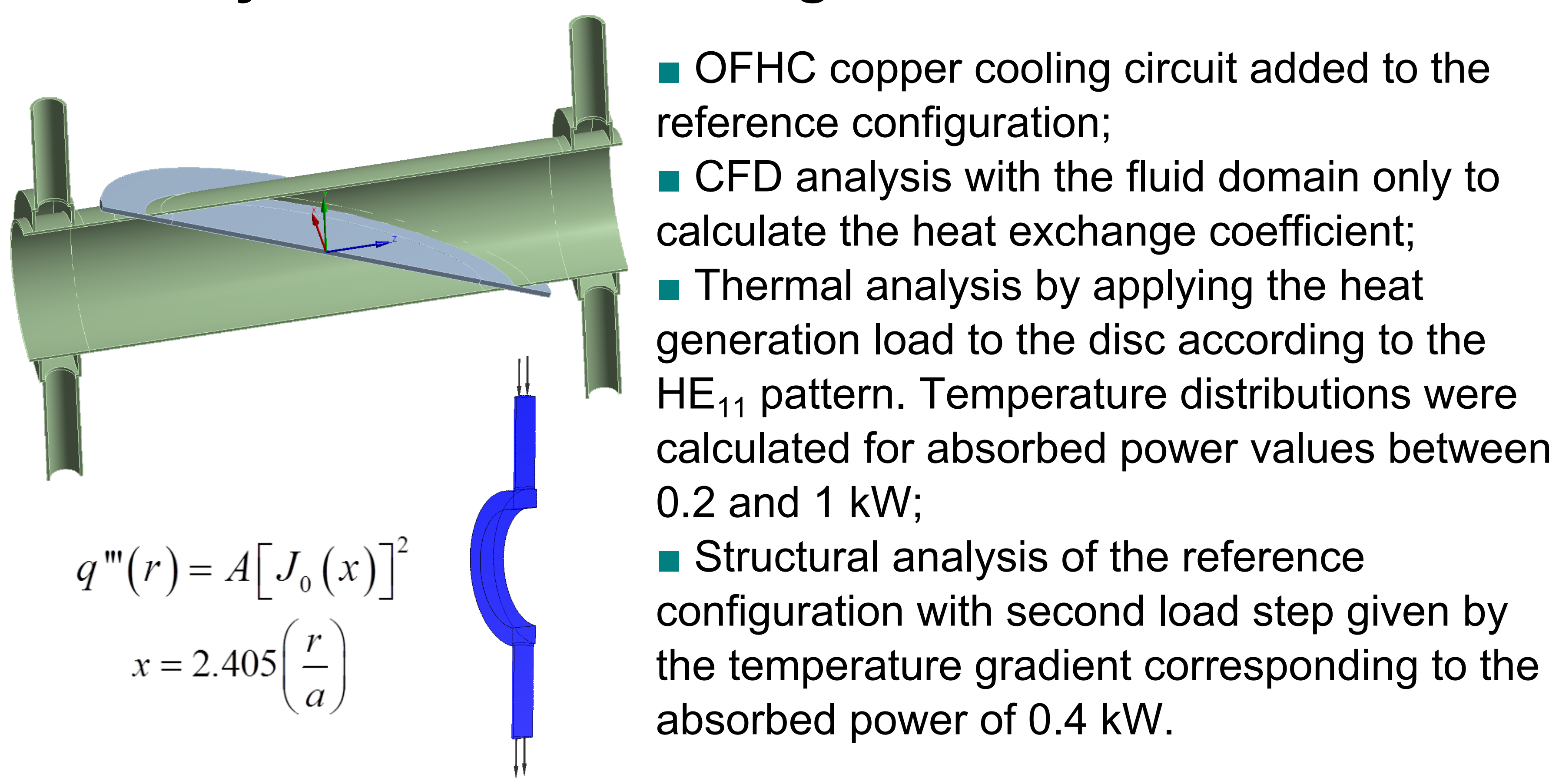

\section{Results}

Reference configuration
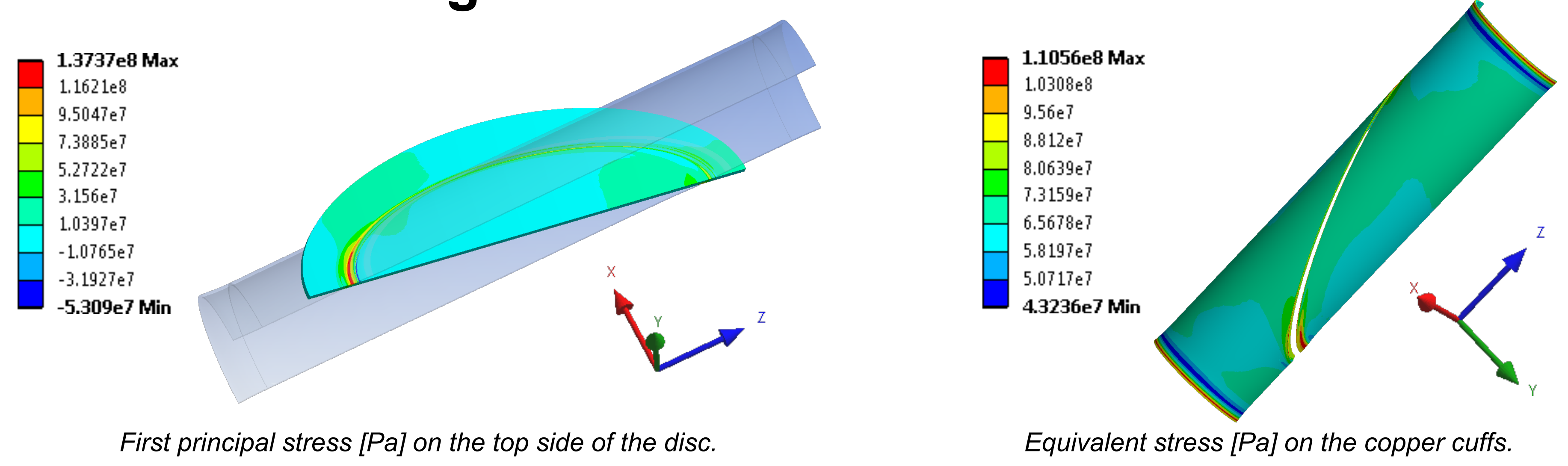

Geometric and constraint configurations
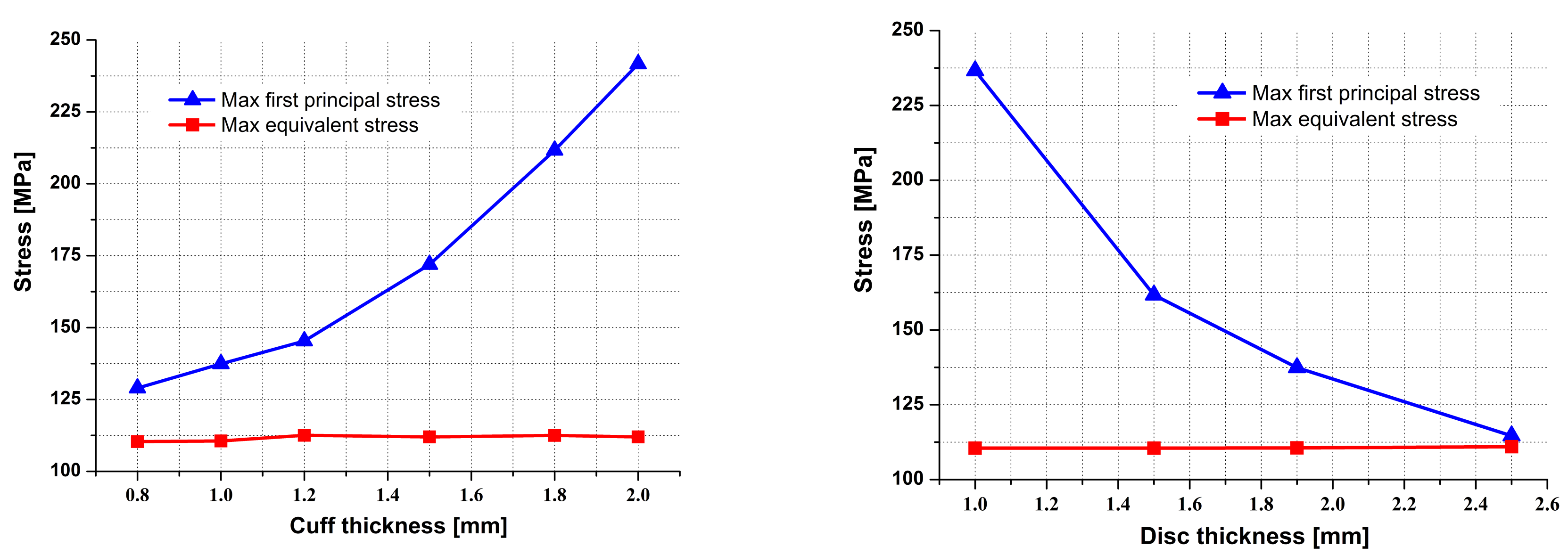

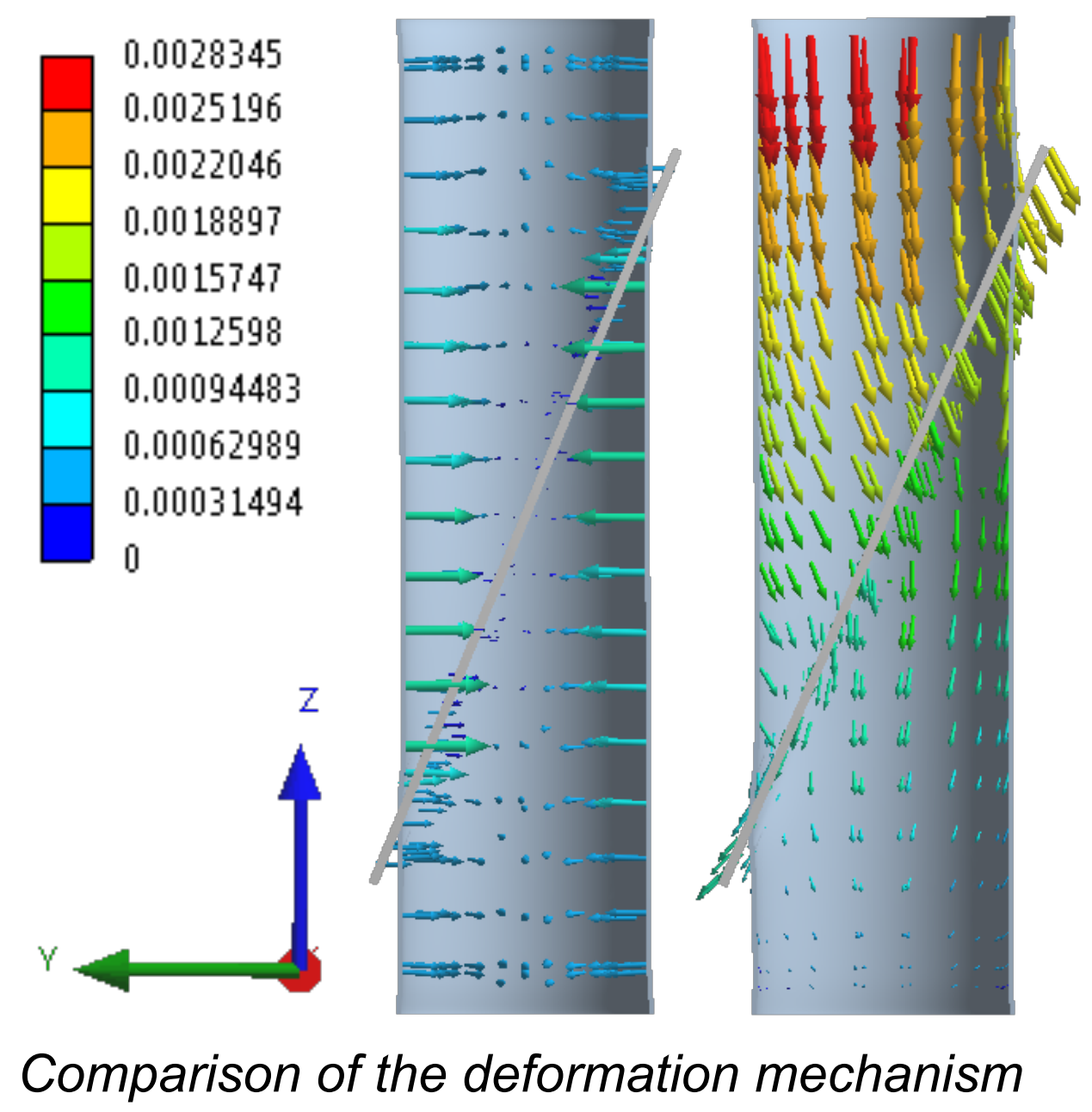

Comparison of the deformation mechanis [m] between reference conf(f)
and configuration 1 (right).

Indirectly water-cooled configuration
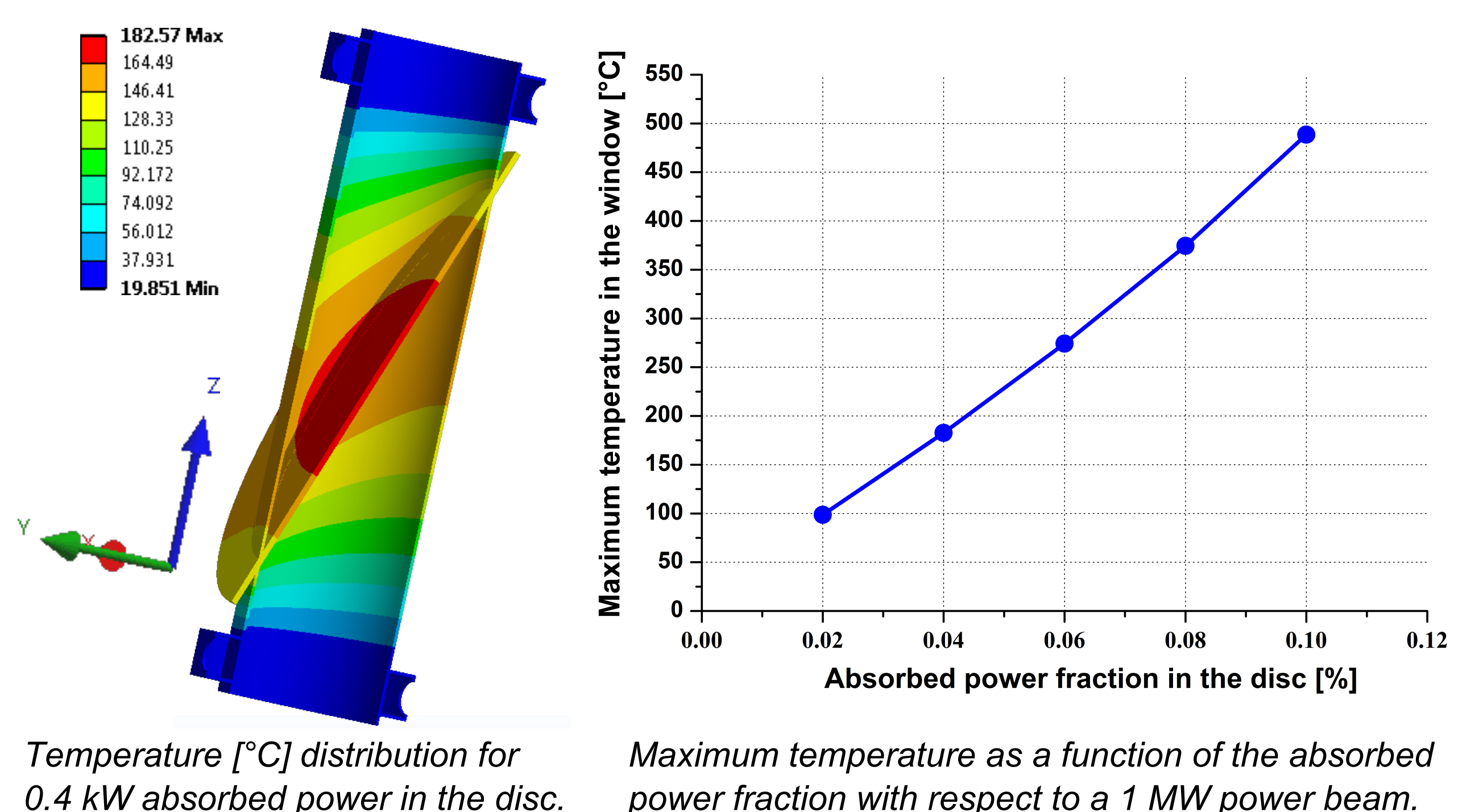

Conclusions

- FEM structural analyses have shown that the CVD diamond Brewster window is a feasible broadband window solution for H\&CD applications. In the reference configuration, the maximum first principal stress is below the permissible stress of diamond which is $150 \mathrm{MPa}$ (ultimate stress is $450-500 \mathrm{MPa}$ ).

- Values of cuff thickness greater than $1.2 \mathrm{~mm}$ and of disc thickness less than $1.7 \mathrm{~mm}$ are not recommended.

- The aspect ratio of the disc affects the first principal stress by less than $3 \%$.

- The configurations 1 and 2 lead to stress values in the disc higher than those obtained in the reference configuration and above the permissible stress.

- The stress field generated in the disc by the cool down phase of the brazing process and the applied temperature gradient is well below the permissible stress. 